\title{
Reducing Surgical Site Infections in Medical Facility Serving the Underserved Population: Curriculum and Brief Review of the Literature
}

\author{
Chukwuemeka Mbagwuํ, Rolanda Willacy ${ }^{2 *}$, Carl-Henri Monfiston², Charles Adebayo ${ }^{2}$ and Robert Wilson² \\ ${ }^{1}$ Howard University College of Medicine, USA
}

${ }^{2}$ Department of Orthopaedic Surgery, Howard University Hospital, USA

*Corresponding author: Rolanda Willacy, Department of Orthopaedic Surgery, Howard University Hospital, USA

Published: 監April 23, 2021

\section{Introduction}

Surgical site infections (SSIs) are a preventable cause of increased morbidity and mortality. They comprise approximately $20 \%$ of all hospital-associated infections (HAIs) and are the secondmost common type of HAIs in the United States [1]. A previous study showed that SSIs were the most common nosocomial infection in surgical patients and were responsible for 38\% of all infections within that category [2]. Surgical site infections lead to prolonged hospital stays, adjunctive procedures, and additive costs [3]. SSI rates are an indicator of the quality of surgical and postoperative care, which calls for the need of increased surveillance systems for these infections.

\section{Patient Dependent Versus Surgeon Dependent}

The literature demonstrates that many, if not all, surgical specialties have made focused efforts aimed at better understanding the preventative measures associated with reducing SSI risk factors. Abdominal (Liver and Kidney transplant, GI, Colon, Liver, or Pancreatic surgery), Thoracic (Heart transplants, CABGs, and Thoracic surgery), Neurosurgical (Ventricular shunts, spinal fusion, Craniotomy), and Cervical (Neck, thyroid, or parathyroid surgeries) procedures all suffer from higher risks of SSIs [4]. While all risk factors analyzed were presented by their temporal association within the surgical process (pre-, intra-, post-, or perioperative), we believe categorization by Patient/Provider-perspective in addition to time course provides greater precision. Classification by Patient-centered risk factors as compared to Provider/Surgeoncentered [CA1] risk factors highlight the important strategies and risk factors associated with the complete surgical time course while also delineating actionable strategies. Multiple national guidelines including the Center for Disease Control's (CDC-US), National Institute for Health and Care Excellence's (NICE-UK), National Health and Medical Research Council's (NHMRC-AUS), and Society for Healthcare Epidemiology of America/Infectious Diseases
Society of America (SHEA/IDSA-US) provide clinical guidelines aligning with patient and provider-centered strategies.

\section{Patient-Centered}

Risk factors for SSI in this classification can be described as modifiable or unmodifiable (Garner 2016) [5]. Age is the most easily distinguished unmodifiable factor, having a direct relationship with an increased risk of SSIs, [6] that is, however, potentially due to comorbidities associated with patient [5]. Patient-centered selfdirected modifiable factors that can impact progression through SSI time course include glucose and Diabetes Mellitus (DM) management, obesity, smoking status, nutritional plan. Glycemic control in patients with DM is associated with improved or adverse outcomes dependent upon the management. Regarding SSIs, glucose levels above $200 \mathrm{mg} / \mathrm{dl}$ have been associated with increased SSIs rates $[7,8]$. As the prevalence of diabetes and prediabetes continues to rise in the U.S., it is necessary to consider Obesity as a significant risk factor since there is significant association between DM and SSI that is consistent across multiple types of surgeries [9] Smoking cessation of at least 30 days prior to operation was included under Category 1B recommendation class by the CDC in their 1999 Guideline for Prevention of Surgical Site Infection [10]. The 1B class is the second highest recommendation category supported by some experimental, clinical, or epidemiological studies and strong theoretical rationale. Smoking cessation/tobacco use/nicotine is not included in the most updated guidelines, [11] but studies continue to show the association between smoking and SSI risk $[12,13]$. Preoperative Nutrition status of patients is important as malnutrition will negatively impact a patient's ability to heal, [14] and lead to increased incidence of SSIs in patients [15].

Patient-centered modifiable factors demanding provider guidance in place of patient's health literacy include preoperative hospitalization stay, antibiotics prophylaxis, patient skin- 
prepping, and inspired $\mathrm{O}_{2}$ content. Increased time in the hospital is associated with increased risk of encountering pathogens and subsequent Hospital-acquired infections, an association that holds for SSIs. Keeping pre-operative hospitalization stay limited as possible is associated with lessened likelihood of SSI incidence [16]. There is consensus amongst multiple guidelines and cohort studies regarding antimicrobial (and more specifically antibiotics) prophylaxis administration when indicated is essential to reducing SSIs [5-20]. There is similar need for further studies to provide consensus on high inspired oxygen therapy towards the reduction of SSI. Recent meta-analyses suggest that the administration of high inspired oxygen fraction $\left(\mathrm{FIO}_{2}\right)$ reduces the risk of SSIs [21]. Data shows statistical significance of reduced SSI rates in hyperoxia group as compared to control groups, however clinical significance still is to be determined, further highlighted by guidelines regarding $\mathrm{FIO}_{2}$ utilization as unresolved. $[5,11]$.

\section{Provider Centered}

Provider-centered risk factors for SSI can be described as procedural or surgeon-related. Such factors are attributed to surgeon experience and ability to control aspects of the operation related to environment, participants, and patient management during the operation. Measurement and feedback of rates of SSI reveals the optimal to suboptimal care provided. Common factors in literature included antiseptic prophylaxis of surgical staff, operating room traffic, patient homeostasis or Normothermia, Clean air, site shaving/hair removal, and surgical attire. Antiseptic prophylaxis including full body bathing or showering and betadine/chlorhexidine utilization towards overall skin-prepping has been included in guidelines as safe and efficacious strategy to preventing SSIs $[11,22]$. It should be stated that within skinprepping, recent systematic reviews state the effectiveness of preoperative bathing with $4 \%$ chlorhexidine towards the reduction of SSI rate in clean surgeries cannot be confirmed. [23] Specific recommendations exist regarding OR parameters include OR entry and ventilation to reduce the risk of pathogens entering the sterile space once surgery begins. A gap in consensus currently exists, as described by Merollini, et al. due to the guidelines recommending ultraclean and laminar flow ORs being outdated and published prior to the more recent contradictory studies suggesting such OR additions were not cost efficient or significant for clinical outcomes [24]. Further studies exploring individual aspects towards OR environment that limit confounding variables are needed. Normothermia, while recommended by CDC and NICE only as much to maintain perioperative normal body temperature, there is a gap in information speaking to strategies to achieve and maintain normothermia, boundaries of normothermia, or the optimal timing and duration of normothermia to prevent SSI $[11,25]$. Sterile gown and surgical glove utilization is recommended for limiting SSIs [24]. Preoperative site shaving for hair removal is recommended against due to the micro abrasions in patients skin that would allow for pathogen entry [11-27]. Exception occurs if the hair will prevent or interfere with the surgical procedure. [5, 27] Surgical attire (scrubs, gowns, headgear, gloves) have demonstrated significant improvement in SSI rates when worn correctly. In the absence of surgical antimicrobial prophylaxis, glove perforation is a risk factor for SSI, thus double gloving to decrease perforation, routine glove changing, are therefore encouraged and defined to be efficacious and safe $[28,29]$.

\section{Preoperative Risk Factors}

\section{Nutritional Deficiency}

There are no currently accepted guidelines regarding ideal patient nutritional status before surgery, however, it has been documented that proper nutrition plays a role in mitigating surgical site infections, and other surgical wound complications. In a study by Greene et al analyzing the records of patients who had undergone a total hip arthroplasty (THA) or total knee arthroplasty (TKA), patients with a preoperative lymphocyte count of fewer than 1,500 cells $/ \mathrm{mm}^{3}$, were five times more likely to later develop a major wound complication [30]. In that same study it was found that patients with a serum albumin below $5 \mathrm{~g} / \mathrm{dL}$ were seven times more likely to suffer from wound complications. A study by Jaberi et al found that in 83 patients that needed surgical debridement after a total joint arthroplasty, patients were at increased risk for developing infection if they had an albumin level under $3.5 \mathrm{mg} /$ $\mathrm{dL}$, or an absolute neutrophil count of less than 1,500 cells $/ \mathrm{mm} 3$ or transferrin levels below $200 \mathrm{mg} / \mathrm{Dl}$ [31]. This information supports that protocols aimed at reducing surgical site infections should include preoperative nutritional assessment and patient education. Long before undergoing any surgical procedure, patients should undergo a preoperative complete blood count and blood chemistry analysis, that includes a specialized report on indicators of nutritional status, such as albumin, transferrin and total lymphocyte levels.

\section{Diabetes Mellitus}

Uncontrolled Diabetes Mellitus (defined as blood glucose over $200 \mathrm{mg} / \mathrm{dL}$ or Hemoglobin A1C (HbA1C) greater than 7\%) has long been known to serve as a significant risk factor in the development of postoperative surgical site infection. A retrospective database study conducted by Marchant et al, found that patients with uncontrolled diabetes had significantly higher odds of surgical site infection when compared to patients with controlled diabetes, and non-diabetic patients, reporting an odds ratio of $2.28(\mathrm{P}=0.002$, with $95 \% \mathrm{CI}=1.36$ to 3.81). Jain, et al. Jain et al. also found diabetes to increase SSI rate and delay wound healing. [32-35]. The study proposed that the mechanism for the increased infection rate, is the compromised function of the leukocytes in diabetic patients. With impaired leukocytes unable to destroy invading pathogens, and unable to check the deleterious proliferation of otherwise benign flora, diabetic patients were found to have approximately a 2.5 times higher risk for SSI than nondiabetics. There is a substantial amount of evidence to support the guideline that very stringent glycemic control should be established well before surgery, especially in diabetic patients. 


\section{Obesity}

The negative health implications associated with obesity are extensive and well documented. Included among the many complications of obesity is the increased risk for surgical site infection, or improper wound healing. A study by Meng, et al. showed that spinal surgery patients with a body mass index (BMI) above $30 \mathrm{~kg} / \mathrm{m} 2$ report increased SSI reporting an odds ratio of 2.13 (with $95 \% \mathrm{CI}=1.55$ to 2.93 ) [34]. Obesity is also a complex risk factor in relation to SSI due to its propensity to present with other SSI risk factors as comorbidities, for example diabetes. In a study reviewing over 2800 total knee and total hip replacements, Namba, et al. reported obese patients had an odds ratio between 4.2 to 6.7 times the infection risk seen in non-obese patients. [35]. Obesity also alters the mechanics of healing, due to elevated adiposity, and increased skin turgor, wound dehiscence is a common complication seen in obese patients postoperatively. Measures to get patients in a healthy BMI range prior to surgery must be an integral part of any protocol or curriculum aimed at mitigating surgical site infections.

\section{Intraoperative Risk Factors}

\section{Skin Preparation}

Skin preparation is an important step in reducing bacterial skin count prior to surgery. A study conducted by Gilliam and Nelson compared bacterial skin counts before and after skin preparation, specifically in primary joint arthroplasty, using iodophor pain and scrub in 30 patients and iodophor and alcohol preparation in 30 patients. It was found that both methods led to similar reduction of bacterial colony counts. One significant difference was the ability of the one-step, water-insoluble iodophor and alcohol solution in improving drape adhesion.

Another component to consider in potential bacterial entry into wounds, is drape lift-off, which can lead to infection. A randomized trial conducted at a single-center compared the use of Dura preparation or an iodine scrub for preoperative skin preparation in 176 patients. The rate of contamination of the wound sites were similar, but there was increased drape life-off in the iodine scrub and paint group. A previous study compared bacterial skin counts before and after skin preparation, specifically in primary joint arthroplasty, using iodophor pain and scrub in 30 patients and iodophor and alcohol preparation in 30 patients.

\section{Surgical Gowns and Gloves}

Surgical gowns are used in performing total joint arthroplasties as well as other surgical procedures. However, their role in significantly decreasing infection is still a debatable topic. Ritter conducted a study that assessed the effectiveness of surgical exhaust gowns in comparison to standard surgical gowns. The results demonstrated that the exhaust gowns provided a $38 \%$ decrease in colony forming units. The results however showed no different during the surgical procedures. Surgical gloves are potentially another source of wound contamination. In particular with THAs [37]. In a study conducted by $\mathrm{McCu}$,e et al, 275 outer and inner gloves were cultured following 10 THAs.. It was found that the gloves most frequently contaminated were the ones used exclusively for draping. The recommendation was made that outer gloves should be removed after draping, and throughout the course of the surgical procedure.

\section{Operating Room Configuration and Traffic}

There are still debates regarding the necessity of ultra-clean air vs. laminar air flow in the OR in helping to decrease deep surgical site infections. Ritter demonstrated a 93\% reduction in airborne bacteria surrounding the wound and bacteria on the instrument table, when laminar air was used. It was also reported from this study that $30 \%$ of unused instruments were contaminated, whereas only $1 \%$ of instruments were contaminated when laminar air was utilized [36]. Another factor involved in prevention of surgical site infections was limiting the number of personnel entering and exiting the OR.

\section{Wound Closure}

Surgical wound closure techniques also may have an effect on the rates of infection. A prospective study by Kahn et al., compared wound closure in 187 primary TKAs and THAs using three different methods, skin staples, subcuticular 3.0 suture or 2-octylcyanoacrylate (OCA) [38]. It was found that closure with OCA, led to less wound discharge within the first 24 hours, both in knees and hips. However, it was demonstrated to have prolonged wound discharge in TKAs that were closed using OCA. Skin staples had faster closure times than OCA or sutures. Additionally, there was no difference observed in infection rates between the three groups. Alternative techniques of closing the fascia and skin such as barbed sutures assist with decreasing time for closure. Further study is still needed to evaluate these techniques and their impact on prevention of surgical site infections.

\section{Conclusion}

There is a wealth of research on preventing surgical site infections as a means to decreasing morbidity and mortality. Patientcentered and provider-centered factors have to be considered in developing protocols related to reducing SSIs. Additionally, preoperative and intraoperative factors should also be considered in mitigating the risk of overall surgical outcomes. Future studies should focus on effective methods to implement hospital-wide interventions to decrease the risk of surgical site infections in this patient population.

\section{References}

1. Klevens M, Edwards JR, Richards CL, Horan TC, Gaynes RP, et al. (2007) Estimating health care associated infections and deaths in US hospitals, 2002. Public Health Rep 32 p. : 160-166.

2. Mangram AJ, Horan TC, Pearson ML, Silver LC, Jarvis WR (1999) Guideline for Prevention of Surgical Site Infection, 1999. Centers for Disease Control and Prevention (CDC). Hospital Infection Control Practices Advisory Committee. Am J Infect Control 27(2): 97-132.

3. Whitehouse JD, Friedman ND, Kirkland KB, Richardson WJ, Sexton DJ (2002) The impact of surgical site infections following orthopaedic surgery at a community hospital; and a university hospital: Adverse 
quality of life, excess length of stay, and extra cost. Infect Control Hosp Epidemiol 23: 183-189.

4. https://www.cdc.gov/nhsn/pdfs/pscmanual/9pscssicurrent.pdf.

5. Garner BH, Anderson DJ (2016) Surgical site infections: an update. Infect Dis Clin North Am 30(4): 909- 929.

6. Kaye KS, Schmit K, Pieper C, Richard S, Kathleen F, et al. (2015) The effect of increasing age on the risk of surgical site infection. J Infect Dis 191(7): 1056-1062.

7. Griffin FA (2005) Best-practice protocols: Preventing surgical site infection. NursManage. 36(11): 20, 2-6.

8. Jeon CY, Furuya EY, Berman MF, Larson EL (2012) The role of preoperative and postoperative glucose control in surgical-site infections and mortality. PLoS One 7(9): e45616.

9. Martin ET, Kaye KS, Knott C, Nguyen H, Santarossa M, et al. (2016) Diabetes and risk of surgical site infection: a systematic review and meta-analysis. Infect Control Hosp Epidemiol 37(1): 88-99.

10. Mangram AJ, Horan TC, Pearson ML, Silver LC, Jarvis WR (1999) Guideline for prevention of surgical site infection, 1999: Hospital Infection Con- trol Practices Advisory Committee. Infect Control Hosp Epidemiol 20: 250-278.

11. Berrios Torres SI, Umscheid CA, Bratzler DW, et al. (2017) Centers for Disease Control and Prevention Guideline for the Prevention of Surgical Site Infection, 2017. JAMA Surg 152 p. : 784-791.

12. Sorensen LT, Karlsmark T, Gottrup F (2003) Abstinence from smoking reduces incisional wound infection: a randomized controlled trial. Ann Surg 238(1): 1-5

13. Nolan MB, Martin DP, Thompson R, Schroeder DR, Hanson AC, et al (2017) Association between smoking status, preoperative exhaled carbon monoxide levels, and postoperative surgical site infection in patients undergoing elective surgery. JAMA Surg 152: 476-483.

14. Rai J, Gill SS, Kumar BR (2002) The influence of preoperative nutritional status in wound healing after replacement arthroplasty. Orthopedics 25(4): 417-421.

15. Fukuda Y, Yamamoto K, Hirao M, Nishikawa K, Maeda S, et al. (2015) Prevalence of malnutrition among gastric cancer patients undergoing gastrectomy and optimal preoperative nutritional support for preventing surgical site infections. Ann Surg Oncol 22 p. : 778-785.

16. H Cheng, BPH Chen, IM Soleas, Nicole CF, Chris GC, al. (2017) Prolonged operative duration increases risk of surgical site infections: a systematic review. Surg Infect (Larchmt) 18(6) : 722-735

17. Ariyan S, Martin J, Lal A, Cheng D, Borah GL, et al. (2015) Antibiotic prophylaxis for preventing surgical-site infection in plastic surgery: an evidence-based consensus conference statement from the American Association of Plastic Surgeons. Plastic and Reconstructive Surgery 135(6): 1723-1739.

18. Astagneau P, FL 'Hériteau, F Daniel, P Parneix, AG Venier, et al. (2009) Reducing surgical site infection incidence through a network: results from the French ISO-RAISIN surveillance system. Journal of Hospital

Infection 72(2): 127-134.

19. Daines BK, Dennis DA, Amann S (2015) Infection prevention in total knee arthroplasty. J Am Acad Orthop Surg 23(6): 356-364.

20. Charles EE, Maureen S (2014) Patient Care Interventions to Reduce the Risk of Surgical Site Infections. AORN J 100(6): 590-602.
21. Yang W, Liu Y, Zhang Y, Zhao QH, He SF (2016) Effect of intra-operative high inspired oxygen fraction on surgical site infection: a meta-analysis of randomized controlled trials. J Hosp Infect 93(4): 329-338.

22. Edmiston CE, Benjamin B, Maria CR, Cindy H, Mary BG, et al. (2013) Reducing the risk of surgical site infec ons: Does chlorhexidine gluconate provide a risk reduc on benefit? Am J Infect Control 41(5): S49-S55.

23. Franco LM, Cota GF, Pinto TS (2017) Preoperative bathing of the surgical site withchlorhexidine for infection prevention: systematic review with meta-analysis. Am J Infect Control 45(4): 343-349.

24. Merollini KM, Zheng H, Graves N (2013) Most relevant strategies for preventing surgical site infection after total hip arthroplasty: guideline recommendations and expert opinion. Am J Infect Control 41(3): 221226.

25. Hypothermia: prevention and management in adults having surgery Paul Crosland 2016

26. Surgical Site Infections 2013.

27. Ilker Uçkay, Stephan Harbarth, Robin Peter, Daniel Lew, Pierre Hoffmeyer, Didier, et al. (2009) Preventing surgical site infection. Where now? J Hosp Infect 8(6): 657-670.

28. McHugh SM, Corrigan MA, Hill AD, Humphreys H (2014) Surgical attire, practices and their perception in the prevention of surgical site infection. Surgeon 12(1): 47-52.

29. Misteli H, Weber WP, Reck S, Rachel R, Marcel Z, et al. (2009) Surgical glove perforation and the risk of surgical site infection. Arch Surg 144(6): 553-558.

30. Greene KA, Wilde AH, Stulberg BN (1991) Preoperative nutritional status of total joint patients: Relationship to postoperative wound complications. J Arthroplasty 6(4): 321-325.

31. Jaberi FM, Parvizi J, Haytmanek CT, Joshi A, Purtill J (2008) Procrastination of wound drainage and malnutrition affect the outcome of joint arthroplasty. Clin Orthop Relat Res 466(6): 1368-1371.

32. Marchant MH, Viens NA, Cook C, Vail TP, Bolognesi MP (2009) The impact of glycemic control and diabetes mellitus on perioperative outcomes after total joint arthroplasty. J Bone Joint Surg Am 91(7): 1621-1629.

33. Jain RK, Shukla R, Singh P, Kumar R (2015) Epidemiology and risk factors for surgical site infections in patients requiring orthopedic surgery. European journal of orthopaedic surgery \& traumatology: orthopedie traumatology 25(2): 251-254.

34. Meng F, Cao J, Meng X (2015) Risk factors for surgical site infections following spinal surgery. J Clin Neurosci 22(12): 1862-1866.

35. Namba RS, Paxton L, Fithian DC, Stone ML (2005) Obesity and perioperative morbidity in total hip and total knee arthroplasty patients. J Arthroplasty 20(3): 46-50.

36. MA Ritter (1999) Operating room environment. Clin Orthop Relat Res p. 369: 103.

37. SF McCue, EW Berg, EA Saunders (1981) Efficacy of double-gloving as a barrier to microbial contamination during total joint arthroplasty. J Bone Joint Surg Am,63(5): 811.

38. Khan RJK, Fick D, Yao F, Tang K, Hurworth M, et al. (2006) A comparison of three methods of wound closure following arthroplasty. A prospective, randomised controlled trial. J Bone Joint Surg 88B: 238-242. 
(c) (i) This work is licensed under Creative

To Submit Your Article Click Here: Submit Article

DOI: 10.32474 /OSMOAJ.2021.05.000208

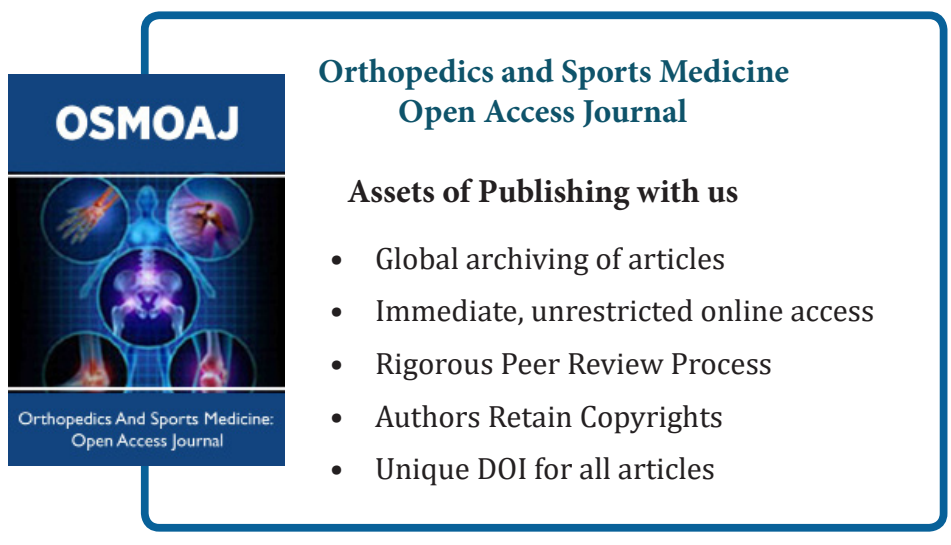

